\title{
Towards Formal Verification of Role-Based Access Control Policies
}

\author{
Somesh Jha, Ninghui Li, Mahesh Tripunitara, Qihua Wang, William Winsborough
}

\begin{abstract}
Specifying and managing access control policies is a challenging problem. We propose to develop formal verification techniques for access control policies to improve the current state of the art of policy specification and management. In this paper, we formalize classes of security analysis problems in the context of Role-Based Access Control. We show that in general these problems are PSPACE-complete. We also study the factors that contribute to the computational complexity by considering a lattice of various subcases of the problem with different restrictions. We show that several subcases remain PSPACE-complete, several further restricted subcases are NP-complete, and identify two subcases that are solvable in polynomial time. We also discuss our experiences and findings from experimentations that use existing formal method tools, such as model checking and logic programming, for addressing these problems.
\end{abstract}

Index Terms - Access control, RBAC, Formal methods, Computational complexity

\section{INTRODUCTION}

Access control is one of the most fundamental and pervasive security mechanisms in use today. The specification and management of access control policies is a challenging problem, and today's administrators have little tools to assist them. As a result, a large number of security breaches are caused by policy misconfigurations. Administrators are often reluctant to change policy settings, as they do not have confidence in whether the resulting policy configurations indeed enforce the policy objectives. The current state of the art of access control policy specification and management is still "what you specify is what you get, but not necessarily what you want". This can be compared to

Somesh Jha: Department of Computer Science, University of Wisconsin at Madison, USA

Ninghui Li and Qihua Wang: Department of Computer Science and CERIAS, Purdue University, Indiana, USA

Mahesh Tripunitara: Motorola Labs, Illinois, USA

William Winsborough: Department of Computer Science, University of Texas at San Antonio, USA software/hardware development before formal verification techniques [7], [27], [28] were developed and successfully deployed. We believe that formal verification techniques for access control policies can be developed to improve the current state of the art.

In almost all access control systems, there is a need to change the authorization state; for example, users and objects are added and removed, users start sharing resources at one moment and stop such sharing later, and users' job functionalities change. This dynamic aspect makes access control particularly challenging. A fundamental problem that deals with the dynamic aspect of access control is safety analysis, which was first formulated by Harrison, Ruzzo, and Ullman [17] for the access matrix model. Safety analysis decides whether undesirable right leakage could occur in future states. Recently, the notion of security analysis, which generalizes safety analysis, was introduced [25], [26]. A Security Analysis Problem instance asks whether an access control system preserves security policy invariants (which encode desired security properties) across state changes. Security analysis also allows the explicit specification of trusted principals. This enables one to ask questions such as: suppose that a set of trusted principals will not initiate any potentially dangerous actions; does a policy invariant hold in all future states? A positive answer provides the assurance that the security of the system depends only on the cooperation of trusted principals.

In this paper (Section II) we study the Security Analysis Problem (SAP) in Role-Based Access Control (RBAC) with the URA97 administrative scheme [37], [38] (we call this problem URASAP). We also describe our experiences building tools for URA-SAP using model checking and logic programming. Our choice of RBAC as the problem domain is motivated by the fact that RBAC [3], [13], [39] is today's most influential access control model. The past decade has seen an explosion of research in 
RBAC. Today, most major information technology vendors are offering products that incorporate some form of RBAC. For example, all major DBMS products support RBAC. Microsoft has brought RBAC to the Windows operating systems by introducing Authorization Manager in Windows Server 2003 [29]. RBAC has also been used in Enterprise Security Management Systems, such as IBM Tivoli Policy Manager [20] and SAM Jupiter [4], [21].

The goal of our work is to develop techniques to help RBAC administrators precisely understand whom they are trusting for maintaining the desirable security properties, or in other words, who will be able to compromise the security of their system.

The contributions of this paper are as follows:

- In Section III, we show that in general URASAP is PSPACE-complete. We also study the factors that contribute to the computational complexity by considering a lattice of various subcases of the problem with different restrictions. We show that several subcases remain PSPACE-complete, several further restricted subcases are NP-complete, and we identify two subcases that are solvable in polynomial time. We observe that the administrative scheme implemented in Oracle's RBAC system falls into one of the two tractable subcases.

- In Section IV, we compare two approaches to using existing tools to perform URASAP and report our findings. One approach is to use model checking (specifically, the tool NuSMV [32]); the other is to use logic programming (specifically, the language XSB [15]).

We discuss related work in Section $\mathrm{V}$ and conclude in Section VI.

\section{Problem Definitions}

In this section, we give precise problem definitions for SAP. We also describe the URA97 RBAC scheme and present the special cases of SAP for the scheme.

\section{A. Access Control Schemes}

In existing work on security analysis in access control systems [25], [26], an access control scheme is defined as a state-transition system $\langle\Gamma, Q, \vdash, \Psi\rangle$, in which $\Gamma$ is a set of states, $Q$ is a set of queries, $\Psi$ is a set of state-transition rules, and $\vdash: \Gamma \times Q \rightarrow$
$\{$ true, false $\}$ determines whether a query in $Q$ is true or not in a given state in $\Gamma$. Each $\psi \in \Psi$ is viewed abstractly as a binary relation on $\Gamma$, i.e., $\psi \subseteq \Gamma \times \Gamma$; it determines whether one state can immediately reach another state. Such a definition abstracts state transition as a binary relation, and does not make explicit which principals initiate a particular action to effect a state transition. As a result, to consider multiple SAP instances with the same state but different sets of trusted users, one has to change the state-transition rule, which is unnatural. We now give a definition that makes the initiators explicit, avoiding such problems.

Definition 1 (Access Control Schemes): An access control scheme is given by a 6-tuple $\langle\Gamma, Q, \vdash$ $, \mathcal{A}, \Sigma, \Psi\rangle$, where $\Gamma$ is a set of states, $Q$ is a set of queries, $\vdash: \Gamma \times Q \rightarrow\{$ true, false $\}$ determines whether a query is true or not in a state, $\mathcal{A}$ is a set of principals, $\Sigma$ is a set of actions, and $\Psi$ is a set of state-transition rules.

A state, $\gamma \in \Gamma$, contains all the information necessary to make access control decisions at a given time. When a query, $q \in Q$, arises from an access request, $\gamma \vdash q$ means that the access corresponding to the request $q$ is granted in the state $\gamma$, and $\gamma \forall q$ means that the access corresponding to $q$ is denied. One may also ask a query that does not correspond to a specific request; for example, one may ask whether every principal that has access to a resource is an employee of an organization. Such queries are useful for understanding the properties of a complex access control system.

Each action $\sigma \in \Sigma$ is a function mapping $\Gamma$ to $\Gamma$. We write $\sigma(\gamma)$ to denote the state that results from applying the action $\sigma$ on the state $\gamma$. Note that $\sigma(\gamma)$ could be $\gamma$; for example, this would happen if the application of the action $\sigma$ on the state $\gamma$ fails. Each action $\sigma$ is associated with a set of principals, denoted by $\operatorname{init}(\sigma)$, i.e., init $(\sigma) \subseteq \mathcal{A}$. Principals in $\operatorname{init}(\sigma)$ are called the initiators of the action; these are the principals that actively carry out the action $\sigma$. In most existing access control schemes, each action is carried out by one initiator, in which case $\operatorname{init}(\sigma)$ is a singleton set. When $\operatorname{init}(\sigma)$ includes two principals $u_{1}$ and $u_{2}$, it means that the active participation of $u_{1}$ and $u_{2}$ is needed to carry out $\sigma$.

Each state-transition rule $\psi \in \Psi$ is given by a subset of $\Sigma$, i.e., $\psi \subseteq \Sigma$. The state transition from $\gamma$ to $\gamma_{1}$ is allowed by $\psi$ (we write $\gamma \rightarrow_{\psi} \gamma_{1}$ ) when there exists an action $\sigma$ in $\psi$ such that $\sigma(\gamma)=\gamma_{1}$. 
Given an access control scheme $\langle\Gamma, Q, \vdash$ , $\mathcal{A}, \Sigma, \Psi\rangle$, an access control system is specified by a pair $(\gamma, \psi)$, where $\gamma \in \Gamma$ is the state of the system and $\psi \in \Psi$ is the state-transition rule that determines which state transitions are allowed.

We say that a set $A$ of principals can take an access control system $(\gamma, \psi)$ to a state $\gamma_{g}$ if principals in $A$ can initiate actions that change the state of the access control system from $\gamma$ to $\gamma_{g}$, i.e., there exists a sequence of actions $\sigma_{1}, \sigma_{2}, \cdots, \sigma_{n}$ such that the following two conditions hold:

1) for each $i$ such that $1 \leq i \leq n$, we have $\sigma_{i} \in \psi$ and $\operatorname{init}\left(\sigma_{i}\right) \subseteq A$.

2) $\sigma_{n}\left(\cdots \sigma_{2}\left(\sigma_{1}(\gamma)\right) \cdots\right)=\gamma_{g}$

Definition 2 (SAP): Given an access control scheme $\langle\Gamma, Q, \vdash, \mathcal{A}, \Sigma, \Psi\rangle$, a security analysis problem (SAP) instance is given by a 4-tuple $\left\langle A_{T}, \gamma, \psi, q\right\rangle$, where $A_{T} \subseteq \mathcal{A}$ is a finite set of trusted principals, $(\gamma, \psi)$ defines an access control system, and $q \in Q$ is a query.

The answer to the instance is true if principals other than those in $A_{T}$ can take the access control system $(\gamma, \psi)$ to a state in which $q$ evaluates to true. That is, this instance asks whether there exists a state $\gamma_{g}$ such that principals in the set $\mathcal{A}-A_{T}$ can take $(\gamma, \psi)$ to the state $\gamma_{g}$ and $\gamma_{g} \vdash q$.

In an instance of SAP, $q$ typically encodes an unsafe situation that should never occur; that is, $\neg q$ would be a policy invariant that should always hold.

\section{B. The URA97 RBAC Scheme}

We now define the access control scheme that we study in this paper, the URA97 RBAC scheme, which is based on the ARBAC97 administrative scheme for RBAC [37], [38]. To our knowledge, ARBAC97 is the first comprehensive and the most influential administrative model for RBAC.

URA97 is one of the three components of ARBAC97 [38]. The other components of ARBAC97 are PRA97 and RRA97, for administering permission-role assignment/revocation, and the role hierarchy, respectively. In this paper, we study the effect of decentralizing user-role assignment and revocation, and assume that changes to the permission-role assignment relation and the role hierarchy are centralized, i.e, made only by trusted users. In other words, whoever is allowed to make changes to permission-role assignment and the role hierarchy will use security analysis and only make those changes that do not violate desirable security properties.

We assume that there are three countable sets: $\mathcal{U}$ (the set of all possible users), $\mathcal{R}$ (the set of all possible roles), and $\mathcal{P}$ (the set of all possible permissions). While the set of all users in any RBAC state is finite, the set of all users that could be added is potentially unbounded. One can think of $\mathcal{U}$ as the set of all possible user-identifiers in a system.

States $(\Gamma)$ : An $\operatorname{RBAC}$ state $\gamma$ is a 6-tuple $\langle U A, P A, R H, C A, C R, C O\rangle$. We call $U A, P A$, and $R H$ parts of the basic state, and $C A, C R, C O$ parts of the administrative state. The basic state is described below; the administrative state is described when we discuss state transitions.

The user assignment relation $U A \subseteq \mathcal{U} \times \mathcal{R}$ associates users with roles, the permission assignment relation $P A \subseteq \mathcal{R} \times \mathcal{P}$ associates roles with permissions, and the role hierarchy relation $R H \subseteq$ $\mathcal{R} \times \mathcal{R}$ is an irreflexive and acyclic relation over $\mathcal{R}$. We use $\succeq_{R H}$ to denote the partial order induced by $R H$, i.e., the transitive and reflexive closure of $R H$. $r_{1} \succeq_{R H} r_{2}$ means that every user who is authorized for $r_{1}$ is also authorized for $r_{2}$ and every permission that is associated with $r_{2}$ is also associated with $r_{1}$.

Given a state $\gamma$, each user has a set of roles for which the user is authorized. We formalize this by defining for every state $\gamma$ a function authorizedRoles $: \mathcal{U} \rightarrow 2^{\mathcal{R}}$

authorizedRoles $(u)=\left\{r \in \mathcal{R} \mid \exists r_{1} \in \mathcal{R}\right.$

$$
\left.\left[\left(u, r_{1}\right) \in U A \wedge\left(r_{1} \succeq_{R H} r\right)\right]\right\}
$$

When $r \in$ authorizedRoles $(u)$, we say that the user $u$ is authorized for the role $r$, or equivalently, $u$ is a member of $r$. We also define $\operatorname{down}(r)$ to be the set of all roles dominated by $r$ and $u p(r)$ to be the set all roles that dominate $r$ as follows:

$$
\begin{aligned}
& \operatorname{down}(r)=\left\{r^{\prime} \in \mathcal{R} \mid r \succeq_{R H} r^{\prime}\right\} \\
& u p(r)=\left\{r^{\prime} \in \mathcal{R} \mid r^{\prime} \succeq_{R H} r\right\}
\end{aligned}
$$

State Transition: $\mathcal{A}, \Sigma$, and $\Psi$ : We now specify $\mathcal{A}$, $\Sigma$, and $\Psi$, which determine how states may change in the URA97 scheme. $\mathcal{A}$ is defined to be $\mathcal{U}$, the set of all possible users. $\Sigma$ consists of two kinds of actions: assignment and revocation actions. Whether these actions succeed or not when applied in a state depends on the administrative state of $\gamma$, namely $C A, C R$, and $C O$, which we describe below. 
- The relation $C A \subseteq \mathcal{R} \times C \times 2^{\mathcal{R}}$ determines who can assign users to roles and the preconditions these users must satisfy. $C$ is the set of conditions, which are expressions formed using roles, the binary operators $\cap$ and $\cup$, the unary operator $\neg$, and parentheses. A tuple $\left\langle r_{a}, c\right.$, rset $\rangle$ in $C A$ means that members of the role $r_{a}$ can assign any user whose role memberships satisfy the condition $c$, to any role $r \in$ rset. For example, $\left\langle r_{0}, r_{1} \cap r_{2} \cap \neg r_{3},\left\{r_{4}\right\}\right\rangle$ $\in C A$ means that a user that is a member of the role $r_{0}$ is allowed to assign a user that is a member of both $r_{1}$ and $r_{2}$, but is not a member of $r_{3}$, to be a member of $r_{4}$.

- The relation $C R \subseteq \mathcal{R} \times 2^{\mathcal{R}}$ determines who can remove users from roles. That $\left\langle r_{a}\right.$, rset $\rangle \in C R$ means that the members of role $r_{a}$ can remove a user from a role $r \in$ rset. Unlike the relation $C A$, there is no preconditions in the relation $C R$ defined in URA97 [38].

We assume that $C A$ and $C R$ satisfy the property that the administrative roles are not affected by $C A$ and $C R$. The administrative roles are those that appear in the first component of each tuple in $C A$ or $C R$. These roles should not appear in the last component of any $C A$ or $C R$ tuple. This condition is satisfied in URA97, which assumes the existence of a set of administrative roles that is disjoint from the set of normal roles.

- $C O$ is a set of mutually exclusive role constraints. Each constraint in $\mathrm{CO}$ has the form smer $\left\langle\left\{r_{1}, \ldots, r_{m}\right\}, t\right\rangle$ where each $r_{i}$ is a role, and $m$ and $t$ are integers such that $1<t \leq m$. This constraint forbids a user from being a member of $t$ or more roles in $\left\{r_{1}, \ldots, r_{m}\right\}$. We say that a set $R$ of roles satisfies a constraint smer $\left\langle\left\{r_{1}, \ldots, r_{m}\right\}, t\right\rangle$ if and only if $\left|R \cap\left\{r_{1}, \ldots, r_{m}\right\}\right|<t$, where || gives the cardinality of a set.

For example, smer $\left\langle\left\{r_{1}, r_{2}\right\}, 2\right\rangle$ means that no user is allowed to be a member of both $r_{1}$ and $r_{2}$. In an $\operatorname{RBAC}$ state $\gamma$, if $r_{1} \in$ authorizedRoles $(u)$ for a user $u$, then an assignment action that assigns the user $u$ to any role in $u p\left(r_{2}\right)$ would fail because of the constraint.

$\Psi$ consists of a single state-transition rule, $\psi$, which is a set of actions:

$$
\begin{aligned}
\psi= & \left\{\operatorname{assign}\left(u_{a}, u_{t}, r_{t}\right) \mid u_{a}, u_{t} \in \mathcal{U} \wedge r_{t} \in \mathcal{R}\right\} \\
& \cup\left\{\operatorname{revoke}\left(u_{a}, u_{t}, r_{t}\right) \mid u_{a}, u_{t} \in \mathcal{U} \wedge r_{t} \in \mathcal{R}\right\}
\end{aligned}
$$

- An assignment action $\operatorname{assign}\left(u_{a}, u_{t}, r_{t}\right)$ means that the user $u_{a}$ assigns the user $u_{t}$ to the role $r_{t}$. When this action is applied to an RBAC state $\gamma$, it succeeds if and only if the following three conditions hold:

- $\left(u_{t}, r_{t}\right) \notin U A$, i.e., the user $u_{t}$ is not already assigned the role $r_{t}$.

- There exists a tuple $\left\langle r_{a}, c\right.$, rset $\rangle \in C A$ such that $r_{a} \in$ authorizedRoles $\left(u_{a}\right)$, authorizedRoles $\left(u_{t}\right)$ satisfies $c$, and $r_{t} \in$ rset.

- authorizedRoles $\left(u_{t}\right) \cup \operatorname{down}\left(r_{t}\right)$ satisfies every constraint in $C O$, i.e., the new role memberships of $u_{t}$ do not violate any constraint.

The assignment action $\operatorname{assign}\left(u_{a}, u_{t}, r_{t}\right)$ may succeed even when $u_{t}$ is already authorized for $r_{t}$ indirectly through other roles. For example, even when $\left(u_{t}, r_{s}\right) \in U A$ and $r_{s} \succeq_{R H} r_{t}$, $\operatorname{assign}\left(u_{a}, u_{t}, r_{t}\right)$ can succeed. The rationale is that these memberships often represent independent relationships. For example, $\left(u_{t}, r_{s}\right) \in$ $U A$ may represent a shorter-term role assignment for $u_{t}$ because of temporary staff shortages, and $\left(u_{t}, r_{t}\right) \in U A$ may represent a longerterm role assignment; then we want to add $\left(u_{t}, r_{t}\right)$ to $U A$ so that when $\left(u_{t}, r_{s}\right)$ is removed from $U A, u_{t}$ is still authorized for $r_{t}$.

When the assignment action is successfully applied to an RBAC state $\gamma$, the resulting state $\gamma^{\prime}$ differs from $\gamma$ only in the user-role relation. The result of a successful application is $U A^{\prime}=$ $U A \cup\left\{\left(u_{t}, r_{t}\right)\right\}$. When the application is not successful, the state does not change.

- A revocation action is of the form revoke $\left(u_{a}, u_{t}, r_{t}\right)$, which means that the user $u_{a}$ revokes the user $u_{t}$ from the role $r_{t}$. When this action is applied to an RBAC state $\gamma$, it succeeds if and only if the following two conditions hold:

- $\left(u_{t}, r_{t}\right) \in U A$, i.e., the user $u_{t}$ is assigned to the role $r_{t}$.

- There exists a tuple $\left\langle r_{a}, r s e t\right\rangle \in C R$ such that $r_{a} \in$ authorizedRoles $\left(u_{a}\right)$, and $r_{t} \in$ rset. 
When the revocation action is successfully applied to an RBAC state $\gamma$, the resulting state $\gamma^{\prime}$ differs from $\gamma$ only in the user-role relation. The result of a successful application is $U A^{\prime}=$ $U A-\left\{\left(u_{t}, r_{t}\right)\right\}$. When the application is not successful, the state does not change.

\section{SAP in URA97}

Definition 3 (URA-SAP): A URA-SAP instance is given by an RBAC state $\gamma=\langle U A, P A, R H$, $C A, C R, C O\rangle$, a set $A_{T} \subseteq \mathcal{U}$ of trusted users, and a query.

We deliberately leave the syntax for queries unspecified in the above definition. Different kinds of queries may be needed for different policy analyses. The simplest kind is to ask whether a user $u$ is a member of a role $r$. More sophisticated queries may ask whether a user's role membership satisfy a condition (e.g., $r_{1} \cup\left(r_{2} \cap \neg r_{3}\right)$ ), or whether the set of members of one role is a subset of the set of members of another role.

An important observation is that the simplest query which asks whether a user is a member of a role can be used to handle several other kinds of queries. For example, if one wants to know whether the system can reach a state in which $u$ 's role membership includes a set $\left\{r_{1}, r_{2}\right\}$ and excludes $\left\{r_{3}\right\}$, one can add a new user $u_{a}$, two new roles $r_{a}$ and $r_{t}$, a user assignment $\left(u_{a}, r_{a}\right)$, and a new tuple $\left(r_{a},\left(r_{1} \cap r_{2} \cap \neg r_{3}\right),\left\{r_{t}\right\}\right)$ to $C A$, and use $u \in r_{t}$ as the query. Similarly, if one wants to know whether the system can go to a state in which $u$ possesses a certain set of permissions, one can compute the role condition that is necessary and sufficient to have the permissions and then translate that into a query about a single role.

Definition 4 (URA-RC-SAP): A URA-RC-SAP instance is a special case of URA-SAP in which a query has the form $u \in r$.

\section{An Example}

Figure 1 shows a simplified role-hierarchy in a bank. This example is inspired by a case-study of a commercial bank that appears in the literature [40]. The bank has two functional roles, Loan Officer and Cashier, apart from the basic Employee role. The bank requires that an employee be a member of exactly one functional role. We quote from [40]: "Ideally, each employee is assigned to one role.".
This is easily achieved using the mutually exclusive role constraint smer $\langle\{$ Loan Officer, Cashier $\}, 2\rangle$.

In our example, the bank allows employees to be reassigned to a different functional role. However, such a reassignment must involve at least two administrators. This results in a separation of privilege in that no single administrator can change a user's functional role by himself.

Consider the example query $q_{b a n k}$ of the form "Bob $\in$ Cashier" where (Bob, Loan Officer) $\in$ $U A_{\gamma}$. With this query, we seek to verify that the bank's policy is indeed satisfied for a user $B o b$ that is a loan officer. If we consider a URA-RCSAP instance with the set of trusted principals $A_{T}=\{$ Alice, Adam $\}$, we observe that the instance is false. The reason is that for $B o b$ to be assigned to Cashier, he must first be revoked from Loan Officer. Otherwise, the entry in $C O_{b a n k}$ would be violated. The only administrator that can revoke $B o b$ from Loan Officer is Adam. However, as Adam is considered to be a trusted principal in the analysis instance, he cannot initiate any administrative actions.

It turns out in our example that the bank's policy is indeed satisfied for any user, even for one that is not (yet) an employee of the bank. This can be verified by running analysis instances with appropriately instantiated parameters. As an example to demonstrate that a user can indeed be reassigned to a different functional role, if the user Carl is a member of Cashier in $U A_{\gamma}$, then a URA-RC-SAP instance with $A_{T}=\emptyset$ and query "Carl $\in$ Loan Officer" is true because Carl can first be revoked from Cashier by $A n d y$, then assigned to Employee by Alice and finally assigned to Loan Officer by Adam. In this case, the cooperation of all three administrators is required.

\section{Computational Complexity}

In this section we study the computational complexity of URA-SAP. In particular, we show that URA-RC-SAP is PSPACE-complete. The main source of the complexity of SAP is that the state space that needs to be explored is potentially large. We would like to understand how different features in URA97 affect this search space; therefore, we consider special cases of URA-SAP that result from restricting the URA scheme in various ways. Answers to the following questions affect the computational complexity of URA-SAP. 


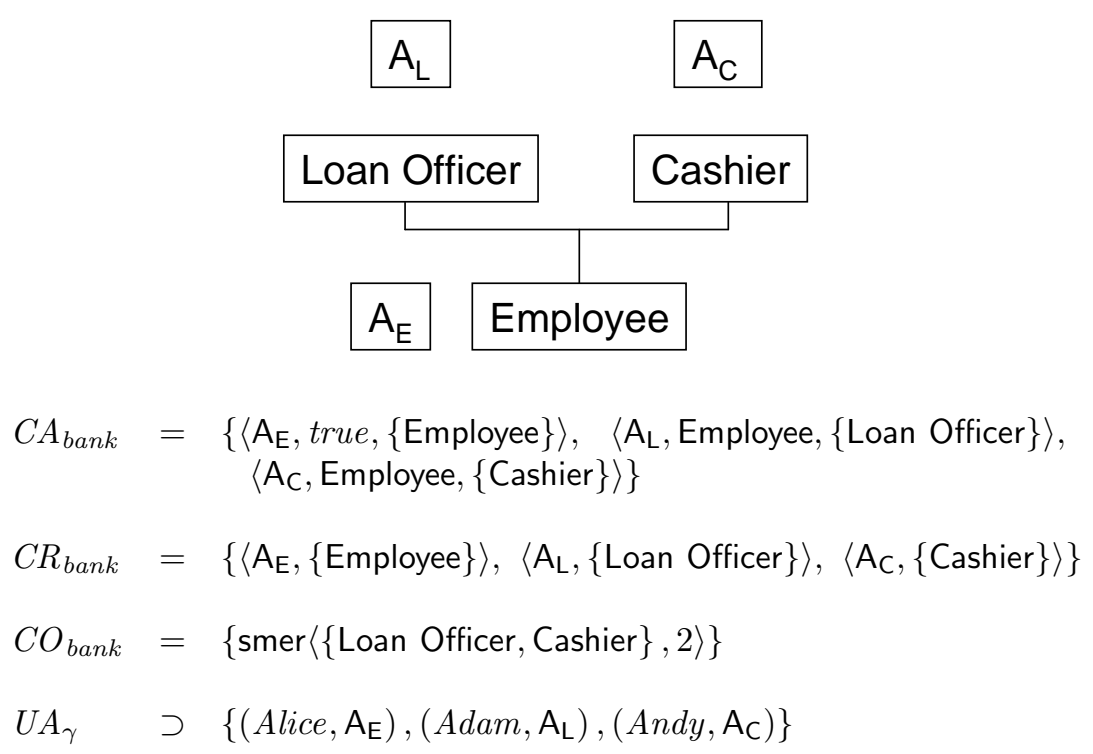

Fig. 1. A simplified view of a bank in which there are two functional roles, Loan Officer and Cashier, both of which inherit from the Employee role. The role hierarchy, $R H_{b a n k}$, is shown in the figure. A different administrative role is associated with each of the roles; $A_{L}$ is for administering Loan Officer, $A_{C}$ for Cashier and $A_{E}$ for Employee. This is reflected in the assignment and revocation rules, $C A_{\text {bank }}$ and $C R_{\text {bank }}$ respectively, for the bank. $C O_{\text {bank }}$ is the set of mutually exclusive role constraints; there is only one constraint, that no user can be both a loan officer and a cashier. The $U A_{\gamma}$ in the figure shows that a different administrative user is assigned to each of the administrative roles.

- What queries are considered? If queries are allowed to contain conjunctions and disjunctions of roles, then URA-SAP is likely to be intractable. For example, in [26], one can pose a query that asks whether the set of users who satisfy $\left(\left(r_{1} \cup r_{2}\right) \cap r_{3}\right)$ is always a subset of the set of users that satisfy $\left(\left(r_{1} \cup r_{2}\right) \cap\left(r_{2} \cup r_{3}\right)\right)$. The intractability results in [26] are consequences of the fact that these sophisticated queries can encode propositional formulas to show NP-hardness. In other words, [26] deals with sophisticated queries but very simple state transition rules.

In this section, in contrast to the work in [26], we focus on the simplest kind of queries, i.e., whether a user $u$ is a member of a role $r$, to better understand the complexity caused by state-transition features within URA97. In other words, we consider URA-RC-SAP.

- Do the preconditions involve only conjunctions? Each tuple in $C A$ has a precondition. It is conceivable that if the precondition involves arbitrary conjunction, disjunction, and negation of roles, then this could make the problem intractable; however, such a result would be less insightful and of less practical interest. In practical systems, one would not expect the precondition to be a very complicated logical formula. In this paper we focus on the special case in which each precondition is a conjunction of roles or their negations. We show below that, for the general case, whether to allow disjunctions in the preconditions or not does not affect the computational complexity.

- Is negation allowed in preconditions in CA? When preconditions in $C A$ may contain negation, one needs to consider the revocation of a users' role memberships in order to satisfy the precondition and be assigned to a new role.

- Are SMER constraints allowed, i.e., is $C O=$ \{\} ? When constraints are allowed, one may need to consider revocations in order to assign a user to a new role.

- Are revocations allowed, i.e., whether $C R=$ \{\} ? One may want to consider the special case that role memberships cannot be revoked.

We summarize the variations we consider in this paper in Figure 2. The main results of this paper are stated in the following theorem. These results are also summarized in Figure 3. 


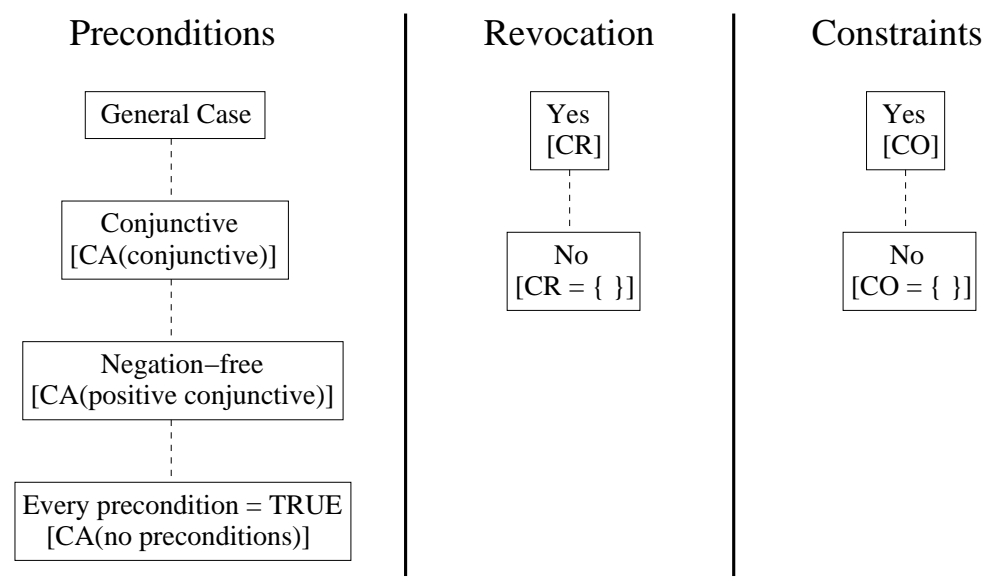

Fig. 2. The possible variations in the features we consider for the preconditions in $C A$, revocation and constraints. A dotted line connects a case with a subcase. For example, negation-free preconditions ([ $C A$ (positive conjunctive)]) is a subcase of conjunctive preconditions ([ $C A$ (conjunctive)]). Various combinations from the three columns are possible. For example, we can consider the analysis problem with negation-free preconditions, with revocation, but without constraints, which corresponds to URA-RC-SAP $C A$ (positive conjunctive), $C R, C O=\{\}]$.

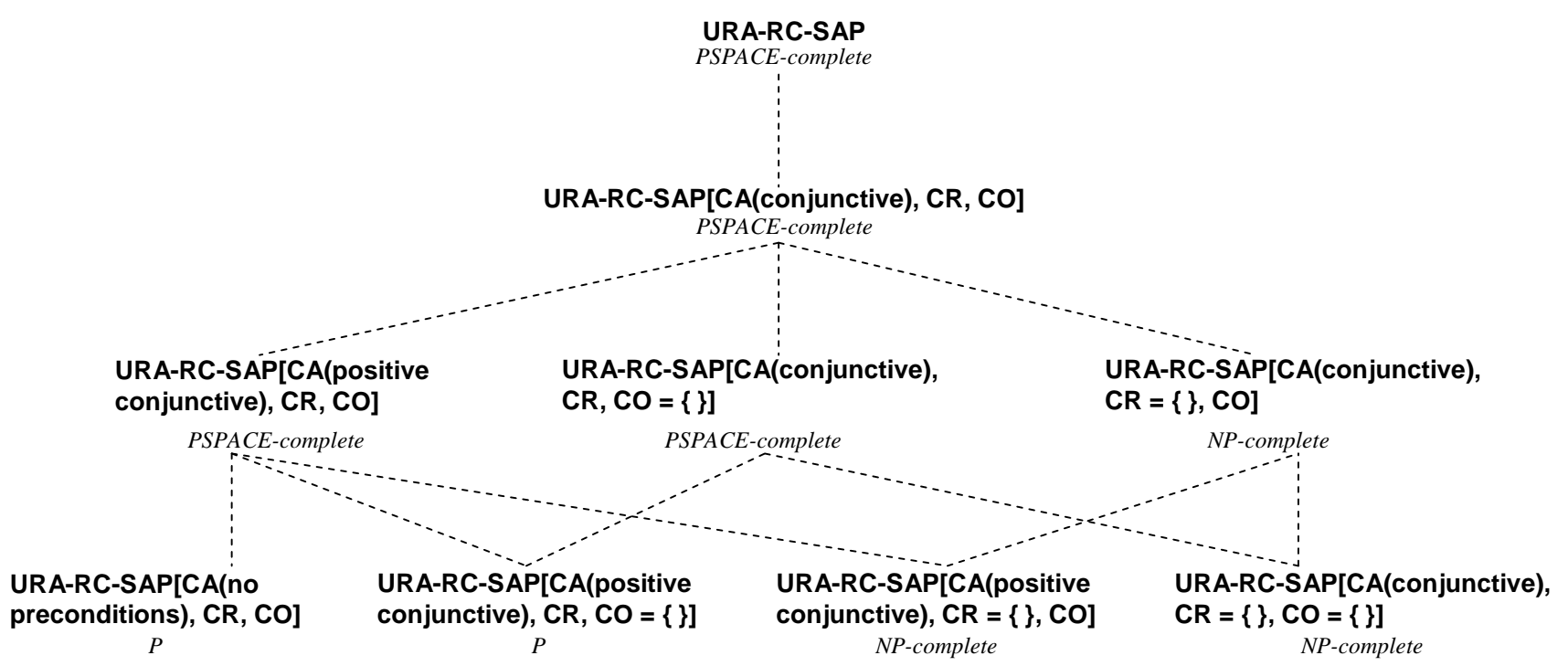

Fig. 3. The summary of computational complexity results for various cases of URA-RC-SAP. A dotted line links a case to a subcase. For example, URA-RC-SAP $C A$ (positive conjunctive), $C R, C O$ ] is a subcase of URA-RC-SAP $C A$ (conjunctive), $C R, C O$ ].

Theorem 1: The computational complexity for URA-RC-SAP and its various subcases are as shown in Figure 3.

Some subcases of the problem are not listed in Figure 3, because they are special cases of the two cases that are known to be in $\mathbf{P}$; thus they are solvable in polynomial time as well. We make several observations from Theorem 1 and Figure 3.

- Whether we allow only conjunctive preconditions or allow arbitrary preconditions does not change the computational complexity of URA-RC-SAP in general. The problem is PSPACE-complete with or without the conjunctive restriction.

- There are three cases in which the problem's complexity changes from PSPACE-complete to NP-complete. All three result from making $C R$ empty. The reason is that if $C R$ is empty, then one needs to consider only role assignments. Any role assignment sequence 
can have length at most polynomial in the size of the problem instance. This makes the problem in NP. On the other hand, there may be exponentially many such possible assignment sequences; thus the problem remains NPcomplete. However, when $C R$ is not empty, the sequence necessary for entering a user into a role may be of exponential length.

- The effect of non-empty $C O$ is identical to the effect of negation in preconditions of $C A$ from the standpoint of computational complexities. The reason is that the effect of a constraint in $C O$ can be "simulated" using negative preconditions in $C A$, and vice versa. We use this fact, for example, in the proofs for Lemmas 4 and 7.

The rest of this section proves the results in Theorem 1.

URA-RC-SAP and three subcases are PSPACE-complete: We first show that the general case of URA-RC-SAP is in PSPACE. We then show that two subcases URA-RC$\mathrm{SAP}[C A$ (positive conjunctive), $C R, C O], \quad$ and URA-RC-SAP $[C A$ (conjunctive), $C R, C O=\{\}]$ are PSPACE-hard. These results together prove the four PSPACE-completeness result in Theorem 1. In Appendix I, we present background information related to Turing machines that we use to establish these results.

Lemma 2: URA-RC-SAP is in PSPACE. Proof: Given a URA-RC-SAP instance, let $\gamma=$ $\langle U A, P A, R H, C A, C R, C O\rangle$, let $U_{T}$ be a set of trusted users, and let $u \in r$ be the query. Notice that the only component of the state that changes is $U A$. Furthermore, we only need to maintain $u$ 's role memberships (the number of roles does not change from the start state). It takes polynomial space to represent the $u$ 's role memberships. Recall that in URA97, administrative roles (i.e., roles that appear in the first component of a tuple in $C A$ and $C R$ ) are not affected by $C A$ and $C R$. Therefore, we do not need to consider memberships of users other than $u$ because these role memberships do not affect whether $u$ can be assigned to a role or not. Observe that in order to determine whether $u$ can be added to a role, the precondition is only about $u$ 's role memberships.

Observe that if we relax the restriction that administrative roles are not affected by $C A$ and $C R$, then we need to maintain role memberships of all users, which can still be done in polynomial space. Therefore, URA-RC-SAP is in PSPACE even without this restriction.

We describe a Non-Deterministic Turing Machine (NDTM) $N M$ to solve this problem. Initially, $N M$ sets initial state $\gamma_{0}$ to be equal to $\gamma$. Given that that $N M$ is in state $\gamma_{i}$, it continues its computation as follows:

- If $\gamma_{i} \vdash q, N M$ stops and outputs yes.

- Assume that $\gamma_{i} \nvdash q$. $N M$ guesses a next state $\gamma_{i+1}$ that changes the user $u$ 's role memberships. $N M$ ensures that such a change conforms to $C A$ and $C R$ and satisfies the constraints in $C O$. NM ensures also that the only users that effect an assign or revoke action are the ones do not belong to $U_{T}$.

The construction given above proves that URARC-SAP is in NSPACE $(O(n))$, where $n$ is the space needed to represent the input URA-RC-SAP instance. Using Savitch's theorem we can conclude that URA-RC-SAP is in DSPACE $\left(O\left(n^{2}\right)\right)$.

Lemma 3: URA-RC-SAP [ $C A$ (positive conjunctive), $C R, C O$ ] is PSPACE-hard.

The proof is given in Appendix II. The proof is by a reduction from the membership problem for linear bounded automata (LBA), which is known to be PSPACE-complete. A LBA is a restricted form of a Turing machine. It differs from a Turing machine in that while the tape is initially considered infinite, only a finite contiguous portion whose length is a linear function of the length of the initial input can be accessed by the read/write head.

Lemma 4: URA-RC-SAP [CA (conjunctive), $C R, C O=\emptyset]$ is PSPACE-hard.

The proof for the above lemma is similar to the proof for Lemma 3. The proof for Lemma 3 uses 2-2 SMER constraints. We simulate the use of such constraints in the proof for Lemma 4 using negative preconditions in $C A$. For example, if a 2-2 SMER constraint smer $\left\langle\left\{r_{1}, r_{2}\right\}, 2\right\rangle$ is used in the proof for Lemma 3 , we can add $\neg r_{1}$ to the precondition of each rule that assigns to $r_{2}$ and add $\neg r_{2}$ to the precondition of each rule that assigns to $r_{1}$.

URA-RC-SAP [ $C A$ (conjunctive), $C R=\{\}, C O$ ] and its two subcases are NP-complete: We first demonstrate that disallowing revocations in URARC-SAP causes the problem to be in NP. We then demonstrate that two subcases are both NP-hard. The first subcase is when we disallow negative pre- 
conditions in $C A$. The second subcase is when we disallow constraints. These results together prove the three NP-completeness results in Theorem 1.

Lemma 5: URA-RC-SAP [CA (conjunctive), $C R=\{\}, C O]$ is in NP.

Proof: We need to demonstrate that if an instance of URA-RC-SAP [ $C A$ (conjunctive), $C R=$ \{\}$, C O]$ is true, then there exists an evidence of size polynomial in the problem that can be efficiently verified. Let the query $q$ in the problem instance be about user $u$ 's membership in the role $r$. As the evidence, we use the shortest state-change sequence from the initial state $\gamma_{0}$ to a state $\gamma_{n}$ such that $\gamma_{n} \vdash q$. Each state-change in this sequence is the assignment of $u$ to a role of which he is not already a member. There can be at most $|R|$ such assignments, where $R$ is the set of roles in the system. (See the proof of Lemma 2 about why we only need to consider assignment of user $u$. Also observe that even if considering assignment of all users, the total number of such assignments is still polynomial in the size of the instance.) Therefore, the state-change sequence is of length at most $|R|$, which is polynomial in the input and can certainly be verified in polynomial time.

Lemma 6: URA-RC-SAP [CA (conjunctive), $C R=\{\}, C O=\{\}]$ is NP-hard.

The proof is in Appendix III; it uses a reduction from the 3SAT problem.

Lemma 7: URA-RC-SAP [ $C A$ (positive conjunctive), $C R=\{\}, C O]$ is NP-hard.

The proof is in Appendix IV. This result should not be surprising given Lemma 6; as discussed earlier, the effects of SMER constraints and negation in preconditions in $C A$ are very similar.

Two subcases that are in P: As we show above, either negation in preconditions or SMER constraints is sufficient to make URA-RC-SAP intractable. However, URA-RC-SAP [ $C A$ (positive conjunctive), $C R, C O=\{\}$ ], that is, when neither negations in preconditions nor SMER constraints are allowed, the problem can be solved in linear time. The reason is that to determine whether $u$ can be a member of a role $r$ in some future state, there is no need to consider revocation as there is no negation in preconditions in $C A$ and there are no SMER constraints. A straightforward quadratic algorithm is to try each tuple in $C A$ and see whether $u$ can be assigned to more roles. As the number of roles that can be assigned according to $C A$ is bounded by the size of $C A$, this algorithm takes at most quadratic time. A linear time algorithm can be obtained by reducing this to the Horn-SAT problem, which can be solved in linear time [10]. Each rule in $C A$ can be viewed as a Horn rule; for example, if one such rule says $r_{1} \cap r_{2}$ is the precondition for $r_{3}$, then this can be translated into a Horn clause " $r_{3} \longleftarrow r 1, r 2$ ". Each initial role membership of the user can be translated into a Horn clause. The query can be translated into a Horn query clause.

Another tractable subcase is URA-RC-SAP [ $C A$ (no preconditions), $C R, C O$ ], which can be solved in quadratic time. In this subcase, every precondition in $C A$ is "true", but we allow revocations and SMER constraints. The algorithm is to first check whether the user $u$ is already a member of the role $r$ (where $u$ and $r$ comprise the query). If not, we revoke $u$ from as many roles as possible using entries from $C R$. We then check whether there exists an entry in $C A$ that we can exercise to cause $u$ to become a member of $r$, while not violating any entry in $C O$. If yes, the algorithm returns "true" and otherwise, it returns "false." This is linear in the sizes of $C A, C R$ and $C O$.

We observe that in some RBAC schemes in practical systems, such as the RBAC scheme in the Oracle database, there is no precondition in role assignment. Security analysis there thus falls under the above tractable case.

\section{TOOLS FOR SECURITY ANALYSIS}

The fact that URA-RC-SAP and several of its subcases are intractable (PSPACE-complete or NP-complete) means that there exist difficult problem instances. In this section, we describe our experiences using logic programming and model checking tools for some realistic instances of URA-RCSAP. Our goals for performing these experiments are two-fold. First, we would like to see whether security analysis instances of nontrivial sizes can be solved in reasonable amounts of time. Our experimental results show that the answer is positive. Second, we would like to compare the effectiveness of model checking and logic programming in security analysis. Our results demonstrate that logic programming outperforms model checking in smaller instances; however, model checking appears to scale better than logic programming. 
The Logic Programming Approach: Logic programming is a declarative, relational style of programming based on first-order logic. A logic program is composed of a set of facts and a number of rules which specify how to derive new facts from known ones. We use XSB [15], a Prologvariant logic-programming system developed at SUNY, Stony Brook. XSB uses SLG resolution [6], which can correctly evaluate many recursive logic programs that would cause SLD-resolution-based Prolog systems to fail to terminate.

Our implementation is a natural reduction from instances of URA-RC-SAP to logic programs. Recall that an instance of URA-RC-SAP consists of an RBAC state $\langle U A, P A, R H, C A, C R, C O\rangle$ and a query of the form $u \in r$, where $u \in \mathcal{U}$ is a user and $r \in \mathcal{R}$ is a role. Our logic program defines a predicate over states that is true when the state is reachable. Each tuple in $C A$ and $C R$ is represented as a rule, while $R H$ and $C O$ are incorporated in the rules representing $C A$ entries. The initial role memberships of the user $u$ is given as a fact, and the evaluation's goal is to find a state in which the answer to the query $u \in r$ is true.

The Model Checking Approach: Model checking is a technique for determining whether a formal model $M$ of a system satisfies a temporal-logic property $p$. A model $M$ can be represented as a 4tuple $\left(S, R, s_{0}, L\right)$, where $S$ is a finite set of states, $R \subseteq(S \times S)$ is a transition relation, $s_{0} \in S$ is an initial state, and $L: S \rightarrow 2^{A P}$ is a labeling of states with propositional formulas from $A P$ (given a state $s, L(s)$ denotes the atomic propositions in $A P$ that are true in $s$ ). We express safety property $p$ in Computation Tree Logic (CTL) with the form AG $f$ (i.e., $p=\mathbf{A G} f$, where $f$ is a formula in propositional logic). (AG $f$ means that always globally the atomic proposition $f$ is true, or in other words $f$ is true in every state reachable from the initial state $s_{0}$.) If the model $M$ satisfies the property $p$, a model checker reports true. If $M$ does not satisfy $p$, a model-checker produces a counter-example that shows an execution that leads to a violation of the property. A thorough treatment of model checking is provided in [7].

The model checker we used is NuSMV [32]. We implemented a program that reads an instance of URA-RC-SAP and then generates a NuSMV program for the instance. Encoding an instance of
URA-RC-SAP as a model in NuSMV is straightforward, e.g., states correspond to user assignments to roles and transitions correspond to rules in $C A$ and $C R$.

Preprocessing: We observe that given a URA-RCSAP instance, many rules in $C A$ and $C R$ may be irrelevant to the query. We use a preprocessing stage to remove these rules. Our experimental data shows that preprocessing can be very effective. Given a query $u \in r$ and an RBAC state, our preprocessing does the following two kinds of pruning:

- Forward pruning: We remove rules that will never be successfully executed. We first compute $R_{l o}$, the set of roles in the initial state that cannot be revoked by rules in $C R$. We then compute $R_{u p}$, the set of roles that may be assigned to the user $u$, and $A$, the set of assignment rules that may be successfully applied. To do this, we initialize $R_{u p}$ with $I$, the initial set of roles that $u$ is a member of, and $A$ with $\emptyset$. For each assignment rule $\alpha$ in $C A$, if the target role of $\alpha$ (i.e. the last component of $\alpha$ ) is not in $R_{l o}$, the positive precondition of $\alpha$ is satisfied by $R_{u p}$, and the negative precondition of $\alpha$ does not contain any role in $R_{l o}$, we add the target role of $\alpha$ to $R_{u p}$ and add $\alpha$ to $A$. We repeat this process of iterating through $C A$ until $R_{u p}$ does not grow. Only assignment rules in $A$ and revocation rules that revoke roles in $R_{u p}$ are kept after the pruning. Letting $|C A|$ be the number of rules in $C A$, the computation of $R_{u p}$ and $A$ requires $O\left(|C A|^{2}\right)$ rule-consideration steps, because each pass through $C A$ adds at least one $\alpha \in C A$ to $A$ and each such $\alpha$ needs not be considered thereafter.

- Backward pruning: Some roles may be irrelevant to assigning the role $r$ in the query. The backward pruning removes assignment and revocation rules about those roles. We compute two sets of roles: $R_{p o}$ is the set of roles that $r$ positively depends on, and $R_{n e}$ is the set of roles that $r$ negatively depends on. We remove assignment rules that assign roles outside $R_{p o}$ and revocation rules that revoke roles outside $R_{n e} . R_{p o}$ is the smallest set that satisfies the following three conditions: (1) $r \in R_{p o}$; (2) if $r_{p} \in R_{p o}$, then any role that dominates $r_{p}$ is also in $R_{p o}$; (3) if $r_{p} \in R_{p o}$, then any role that appears in the positive precondition of a 
$C A$ entry assigning to $r_{p}$ is also in $R_{p o}$. $R_{n e}$ is the smallest set that satisfies the following conditions: (1) if $r_{p} \in R_{p o}$, then any role that appears (or dominates a role that appears) in the negative precondition of a $C A$ entry assigning to $r_{p}$ is in $R_{n e}$; (2) if $r_{p} \in R_{p o}$, then any role that is (or dominates a role that is) mutually exclusive with $r_{p}$ is in $R_{n e}$.

The preprocessing takes time at most cubic in the size of the URA-RC-SAP instance.

Experimental results: We performed experiments using two kinds of instances. Manually crafted instances are designed to "hide" an unsafe state after a long sequence of transitions. These instances forced the analysis tools to search deep in the state space. Randomly generated instances contain a relatively large number of roles and transition rules.

Experiments were performed on a Windows workstation with an Intel P4 3G CPU and 512M of memory. We tested the performance of NuSMV and XSB on several instances. Table I presents results for seven instances, two of which were manually generated and five of which were randomly generated.

Experimental results show that the number of rules is a crucial factor in determining runtime. Therefore, the preprocessing step plays an important role in improving the efficiency of both logic programming and model checking implementations. Many instances, such as Rand2, that cannot be solved within 30 minutes without preprocessing are solved within a few seconds with preprocessing.

Both XSB and NuSMV are efficient in cases that have a small number of transition rules. For the example in Section II-D, XSB uses 0.016 seconds and NuSMV uses 0.125 seconds. When tested on a manually crafted instance Man2 with 16 roles and 40 rules (29 left after preprocessing) that requires a sequence of at least 22 transitions before reaching an unsafe state, XSB uses 0.05 seconds and NuSMV uses 0.13 seconds. When preprocessing is effective, such as instance Rand2 with 100 roles and 250 rules (20 rules left after preprocessing), XSB uses 0.55 seconds and NuSMV executes in 0.94 seconds.

It appears that using XSB does not scale as well as using NuSMV. For example, when it came to the randomly generated instance Rand5, with 25 roles and 79 rules (57 left after preprocessing), XSB ran out of memory after 17 minutes, while
NuSMV returned with an answer within 73 seconds. An observation is that the runtime of the XSB grows linearly with the number of reachable states. ${ }^{1}$ However, NuSMV uses binary decision diagrams (BDDs) to represent its state space, so its runtime depends on the regularity of the state space. A point worth mentioning is that XSB consumes memory quickly. For the instance Rand4 with 30 roles and 88 rules (27 left after preprocessing), XSB uses more than $400 \mathrm{MB}$ of memory. The high demand on memory impairs the scalability of our XSB program. In contrast, the BDD-based NuSMV requires less memory than XSB.

Both model checking and logic programming have been used in network-vulnerability analysis [18], [41]. Recently, Ou et al. [33] showed that, in the context of network-vulnerability analysis, logic programming is much more scalable than model checking. Our experimentation data show that for URA-RC-SAP scalability of logic programming is worse than model checking. This is because in network vulnerability analysis, one can make the monotonicity assumption, i.e., if an attacker gains a privilege, it never loses it. However, in security analysis, because of negative preconditions and mutual exclusion constraints, the monotonicity assumption does not hold, and one has explore the state space.

In real-word large-scale RBAC systems, even though the number of roles in the whole system may be large, we expect that the roles that are relevant for any given query will be only a small portion of all roles. Therefore, we conjecture that our approach of combining preprocessing with existing tools such as NuSMV will be able to handle many queries.

\section{RELATED WORK}

In their landmark paper [17], Harrison et al. formalized the safety analysis problem in the access matrix model; the problem determines whether a protection system can reach a state in which a particular right is leaked. They show that safety analysis is undecidable in their scheme [17]. Since then, safety analysis has attracted considerable attention in the research community. Safety analysis in monotonic versions of the HRU scheme has been studied in [16]. Jones et al. introduced the

\footnotetext{
${ }^{1}$ The statistics on total states and reachable states in Table I are actually for NuSMV, but the statistics for XSB should be similar.
} 


\begin{tabular}{|c|c|c|c|c|c|c|c|}
\hline & Man1 & Man2 & Rand1 & Rand2 & Rand3 & Rand4 & Rand5 \\
\hline Num. of Roles & 12 & 16 & 15 & 100 & 40 & 30 & 25 \\
\hline Num. of Rules & 31 & 40 & 45 & 250 & 92 & 88 & 79 \\
\hline Num. of Rules $(A P)$ & 22 & 29 & 34 & 20 & 27 & 37 & 57 \\
\hline Transition Length & 15 & 22 & 1 & 2 & 3 & 2 & 6 \\
\hline \hline Total States & $1.70 \mathrm{E}+7$ & $1.76 \mathrm{E}+9$ & $6.60 \mathrm{E}+8$ & NA & NA & NA & NA \\
\hline Reachable States & $1.31 \mathrm{E}+5$ & $2.69 \mathrm{E}+6$ & $7.54 \mathrm{E}+5$ & NA & NA & NA & NA \\
\hline XSB Runtime & $0.55 \mathrm{~s}$ & $14.22 \mathrm{~s}$ & $109.64 \mathrm{~s}$ & NA & NA & NA & NA \\
\hline NuSMV Runtime & $0.188 \mathrm{~s}$ & $1.78 \mathrm{~s}$ & $2.10 \mathrm{~s}$ & NA & NA & NA & NA \\
\hline Total States $(A P)$ & $1.22 \mathrm{E}+7$ & $1.29 \mathrm{E}+9$ & $5.02 \mathrm{E}+8$ & $1.08 \mathrm{E}+49$ & $3.40 \mathrm{E}+20$ & $7.82 \mathrm{E}+15$ & $4.90 \mathrm{E}+13$ \\
\hline Reachable States $(A P)$ & 3456 & 6000 & 6720 & 75264 & $7.31 \mathrm{E}+5$ & $3.11 \mathrm{E}+6$ & $3.98 \mathrm{E}+7$ \\
\hline XSB Runtime $(A P)$ & $0.02 \mathrm{~s}$ & $0.05 \mathrm{~s}$ & $0.06 \mathrm{~s}$ & $0.55 \mathrm{~s}$ & $5.70 \mathrm{~s}$ & $19.94 \mathrm{~s}$ & NA \\
\hline NuSMV Runtime $(A P)$ & $0.11 \mathrm{~s}$ & $0.13 \mathrm{~s}$ & $0.13 \mathrm{~s}$ & $0.94 \mathrm{~s}$ & $1.27 \mathrm{~s}$ & $5.44 \mathrm{~s}$ & $72.94 \mathrm{~s}$ \\
\hline
\end{tabular}

TABLE I

EXPERIMENTAL DATA ON URA-RC-SAP INSTANCES USING XSB AND NUSMV. INSTANCES WITH NAMES BEGINNING

WITH Man WeRE MANUALLY CRAFTED, WHILE THOSE BEGINNING WITH Rand WERE RANDOMLY GENERATED.

STATISTICS ON TOTAL STATES AND REACHABLE STATES IS FOR NUSMV ONLY. ROWS MARKED (AP) PRESENT RESULTS AFTER PREPROCESSING. NA INDICATES THAT, FOR THE NUSMV CASES, THE PROGRAM DID NOT FINISH RUNNING WITHIN 30 MINUTES, OR, FOR THE XSB CASE, RAN OUT OF THE MEMORY.

Take-Grant scheme [19], in which safety can be decided in linear time. Sandhu et al. introduced the Schematic Protection Model [35], the Extended Schematic Protection Model [1], and the Typed Access Matrix model [36]. Budd [5] and Motwani et al. [30] studied grammatical protection systems. Soshi et al. [43] studied safety analysis in the Dynamic Typed Access Matrix model. These models all have subcases where safety is decidable. Solworth and Sloan [42] introduced a discretionary access control model in which safety is decidable. This thread of research has produced many new access control schemes but has had limited impact on access control systems used in practice, probably because the proposed schemes are either too simplistic to be useful or too arcane to be usable. In this paper, we focus on policy analysis problems in RBAC, which was invented not for the purpose of safety analysis, but for meeting the access control need of real-world applications.

Influential works on RBAC include the pioneering work by Ferraiolo et al. [11], [12] and the widely cited RBAC96 family of formal RBAC models developed by Sandhu et al. [39]. Recently, a standard for RBAC has been proposed and adopted as an ANSI Standard [3], [13]. Administration of RBAC is about controlling who can update the various relations in an RBAC system. The most well-known work on administration of RBAC is ARBAC97, developed by Sandhu et al. [37], [38]. Recently,
Crampton and Loizou [9] introduced the notion of administrative scope and an RBAC administration scheme based on it.

An administrative scheme in conjunction with the representation for an RBAC state naturally lends itself to the safety question in RBAC. The work that is closest to this paper is such work on safety and security analysis in RBAC. Li and Tripunitara [26] studied security analysis for two particular RBAC schemes derived from ARBAC97 [38]: AATU (Assignment And Trusted Users) and AAR (Assign And Revocation), both of which are sub-schemes of the URA97 scheme [38]. The main results in [26] are that security analysis in AATU and AAR are intractable (NP-hard) in general, but can be solved in polynomial time for semi-static queries. The intractability results there are consequences of the fact that a query may be able to encode an arbitrary boolean formula. The techniques used to establish tractable results was to reduce the problem to security analysis in the RT family of trust-management languages [25]. We observe that neither AATU nor AAR allows negative preconditions or constraints. We have shown that URA-RC-SAP with these restrictions are solvable in quadratic time and given direct algorithm for solving them. We point out, even though the role-containment queries are special cases of semi-static queries, our two tractable cases do not follow from results in [26], because AATU does not allow revocation and AAR does not allow 
trusted users. In essence, the results in [26] deal with very simple state transition rules but sophisticated queries. In this paper, we consider simple queries, but sophisticated state transitions. Since RT [24] is monotonic, it is unclear how to extend the techniques in [26] to deal with negative preconditions or constraints. Li and Tripunitara [26] explicitly mentioned dealing with negative preconditions and constraints as an open problem.

Koch et al. have proposed an RBAC scheme based on a graph-based formalism [23] and have demonstrated that safety is decidable in a subscheme [22]. However, the decidable fragment of the graph-based formalism [22] does not allow negative application conditions, which are used to specify negative preconditions in assignment rules in the graph-based formalism for RBAC [23]. Therefore, the decidability result applies only to the subcase without negative preconditions or mutual exclusion constraints. Furthermore, in [22], it has only been shown that safety is decidable in this case; no concrete computational complexity result is given in [22]. The proof shows that the search space is finite; however, searching the space likely takes exponential time. We show that for the case that can be modeled in the decidable fragment, namely, without negative precondition or constraints, URARC-SAP is decidable in quadratic time. For cases with negative preconditions and/or constraints, we have given precise computational complexities for them.

Some work related to safety in access control (e.g., [42]) refers to the work by Crampton [8] and Munawer and Sandhu [31] to claim that safety is undecidable in the ARBAC97 scheme. We point out that the undecidability results in Crampton [8] and Munawer and Sandhu [31] are not about the ARBAC97 scheme. The scheme considered by Crampton [8] adds two new features to ARBAC97. One is to allow changes to the $C A$ and $C R$ relations. (Sandhu et al. [38] state specifically that it is assumed that in an ARBAC97 system, these relations are static and may be changed only by (a trusted) chief security officer.) The other is to allow a state-change rule to include an arbitrary command specified using a construct similar to that proposed by Harrison et al. [17]. Such constructs do not exist in ARBAC97. Munawer and Sandhu [31] present a simulation of the Augmented Typed Access Matrix (ATAM) scheme [2] in a particular RBAC scheme that has similar features as those in Crampton [8].

Sasturkar et al. [44] also studied policy analysis problems in ARBAC97-based systems. They established a connection between the analysis problem and planning in artificial intelligence. Our work has a number of differences. First, they showed only that the analysis problem is PSPACE-complete when revocation rules also have preconditions, which are not in the ARBAC97 model. As the PSPACEhardness result is by a reduction from a planning problem, and the reduction requires revocation rules to have preconditions, the results in [44] cannot entail that URA97-RC-SAP is PSPACE-hard. Our proof uses a direct reduction from the membership problem for linear bounded automata (LBA), and our result that URA-RC-SAP is PSPACEcomplete is stronger because it implies the PSPACEcompleteness result in [44] (proving the in PSPACE part is straightforward). Second, in addition to complexity results, we have also developed tools for such analysis using model checking tools and logic programming and have experimentally evaluated the two approaches. Third, the usage of planning in [44] enables the authors to obtain results on a wider class of problems than those studied in this paper. For example, they also consider special cases where the number of literals in a precondition is limited to a small number.

\section{CONCLUSION AND Future Work}

We have formalized classes of security analysis in the context of RBAC. We have shown that URASAP is PSPACE-complete in the general case and that a number of special cases of the problems are NP-complete. We have also shown that model checking is a promising approach to solve these problems. In the future we plan to look at more sophisticated queries and other administration schemes.

\section{REFERENCES}

[1] P. Ammann and R. S. Sandhu. Safety analysis for the extended schematic protection model. In Proceedings of the 1991 IEEE Symposium on Security and Privacy, pages 87-97, May 1991.

[2] P. Ammann and R. S. Sandhu. Implementing transaction control expressions by checking for absence of access rights. In Proceedings of the Eighth Annual Computer Security Applications Conference (ACSAC), Dec. 1992.

[3] ANSI. American national standard for information technology - role based access control. ANSI INCITS 359-2004, Feb. 2004. 
[4] R. Awischus. Role based access control with the security administration manager (SAM). In Proceedings of the second ACM workshop on Role-based access control table of contents (RBAC '97), pages 61-68, 1997.

[5] T. Budd. Safety in grammatical protection systems. International Journal of Computer and Information Sciences, 12(6):413-430, 1983.

[6] W. Chen and D. S. Warren. Tabled evaluation with delaying for general logic programs. Journal of the ACM, 43(1):20-74, Jan. 1996.

[7] E. M. Clarke, O. Grumberg, and D. A. Peled. Model Checking. MIT Press, 2000.

[8] J. Crampton. Authorizations and Antichains. $\mathrm{PhD}$ thesis, Birbeck College, University of London, UK, 2002.

[9] J. Crampton and G. Loizou. Administrative scope: A foundation for role-based administrative models. ACM Transactions on Information and System Security, 6(2):201-231, May 2003.

[10] W. F. Dowling and J. H. Gallier. Linear-time algorithms for testing the satisfiability of propositional horn formulae. Journal of Logic Programming, 1(3):267-284, 1984.

[11] D. F. Ferraiolo, J. A. Cuigini, and D. R. Kuhn. Role-based access control (RBAC): Features and motivations. In Proceedings of the 11th Annual Computer Security Applications Conference (ACSAC'95), Dec. 1995.

[12] D. F. Ferraiolo and D. R. Kuhn. Role-based access control. In Proceedings of the 15th National Information Systems Security Conference, 1992.

[13] D. F. Ferraiolo, R. S. Sandhu, S. Gavrila, D. R. Kuhn, and R. Chandramouli. Proposed NIST standard for role-based access control. ACM Transactions on Information and Systems Security, 4(3):224-274, Aug. 2001.

[14] M. R. Garey and D. J. Johnson. Computers And Intractability: A Guide to the Theory of NP-Completeness. W.H. Freeman and Company, 1979.

[15] T. X. R. Group. The XSB programming system. http://xsb.sourceforge.net/.

[16] M. A. Harrison and W. L. Ruzzo. Monotonic protection systems. In R. A. DeMillo, D. P. Dobkin, A. K. Jones, and R. J. Lipton, editors, Foundations of Secure Computation, pages 461-471. Academic Press, Inc., 1978.

[17] M. A. Harrison, W. L. Ruzzo, and J. D. Ullman. Protection in operating systems. Communications of the ACM, 19(8):461471, Aug. 1976.

[18] S. Jha, O. Sheyner, and J. M. Wing. Two formal analysis of attack graphs. In 15th IEEE Computer Security Foundations Workshop (CSFW-15 2002), June 2002.

[19] A. K. Jones, R. J. Lipton, and L. Snyder. A linear time algorithm for deciding security. In 17th Annual IEEE Symposium on Foundations of Computer Science (FOCS), pages 33-41, October 1976.

[20] G. Karjoth. The authorization model of Tivoli Policy Director. In Proceedings of the 17th Annual Computer Security Applications Conference, pages 319-328, Dec. 2001.

[21] A. Kern. Advanced features for enterprise-wide role-based access control. In Proceedings of the 18th Annual Computer Security Applications Conference, pages 333-343, Dec. 2002.

[22] M. Koch, L. V. Mancini, and F. Parisi-Presicce. Decidability of safety in graph-based models for access control. In Proceedings of the Seventh European Symposium on Research in Computer Security (ESORICS 2002), pages 229-243. Springer, Oct. 2002.

[23] M. Koch, L. V. Mancini, and F. Parisi-Presicce. A graph-based formalism for RBAC. ACM Transactions on Information and System Security, 5(3):332-365, Aug. 2002.

[24] N. Li, J. C. Mitchell, and W. H. Winsborough. Design of a role-based trust management framework. In Proceedings of the
2002 IEEE Symposium on Security and Privacy, pages 114 130. IEEE Computer Society Press, May 2002.

[25] N. Li, J. C. Mitchell, and W. H. Winsborough. Beyond proofof-compliance: Security analysis in trust management. Journal of the ACM, 52(3):474-514, May 2005. Preliminary version appeared in Proceedings of 2003 IEEE Symposium on Security and Privacy.

[26] N. Li and M. V. Tripunitara. Security analysis in role-based access control. In Proceedings of the Ninth ACM Symposium on Access Control Models and Technologies (SACMAT 2004), pages 126-135, June 2004.

[27] Z. Manna and A. Pnueli. The Temporal Logic of Reactive and Concurrent Systems. Springer, 1992.

[28] Z. Manna and A. Pnueli. Temporal Verification of Reactive Systems: Safety. Springer, 1995.

[29] D. McPherson. Role-based access control for multi-tier applications using authorization manager. http://www.microsoft.com/technet/prodtechnol/windowsserver 2003/technologies/management/athmanwp.mspx.

[30] R. Motwani, R. Panigrahy, V. A. Saraswat, and S. Ventkatasubramanian. On the decidability of accessibility problems (extended abstract). In Proceedings of the Thirty-Second Annual ACM Symposium on Theory of Computing, pages 306-315. ACM Press, May 2000.

[31] Q. Munawer and R. S. Sandhu. Simulation of the augmented typed access matrix model (ATAM) using roles. In Proceedings of INFOSECU99 International Conference on Information and Security, 1999.

[32] NuSMV: a new symbolic model checker. http://afrodite.itc.it:1024/ nusmv/.

[33] X. Ou, S. Govindavajhala, and A. W. Appel. MulVAL: A logicbased network security analyzer. In Proceedings of the 14th USENIX Security Symposium, Aug 2005.

[34] C. H. Papadimitriou. Computational Complexity. Addison Wesley Longman, 1994.

[35] R. S. Sandhu. The schematic protection model: Its definition and analysis for acyclic attenuating systems. Journal of the ACM, 35(2):404-432, 1988.

[36] R. S. Sandhu. The typed access matrix model. In Proceedings of the 1992 IEEE Symposium on Security and Privacy, pages 122-136. IEEE Computer Society Press, May 1992.

[37] R. S. Sandhu and V. Bhamidipati. Role-based administration of user-role assignment: The URA97 model and its Oracle implementation. Journal of Computer Security, 7, 1999.

[38] R. S. Sandhu, V. Bhamidipati, and Q. Munawer. The ARBAC97 model for role-based aministration of roles. ACM Transactions on Information and Systems Security, 2(1):105-135, Feb. 1999.

[39] R. S. Sandhu, E. J. Coyne, H. L. Feinstein, and C. E. Youman. Role-based access control models. IEEE Computer, 29(2):3847, February 1996.

[40] A. Schaad, J. Moffett, and J. Jacob. The role-based access control system of a European bank: A case study and discussion. In Proceedings of the Sixth ACM Symposium on Access Control Models and Technologies, pages 3-9. ACM Press, 2001.

[41] O. Sheyner, J. W. Haines, S. Jha, R. Lippmann, and J. M. Wing. Automated generation and analysis of attack graphs. In IEEE Symposium on Security and Privacy, 2002.

[42] J. A. Solworth and R. H. Sloan. A layered design of discretionary access controls with decidable safety properties. In Proceedings of IEEE Symposium on Research in Security and Privacy, May 2004.

[43] M. Soshi. Safety analysis of the dynamic-typed access matrix model. In Proceedings of the Sixth European Symposium on Research in Computer Security (ESORICS 2000), pages 106121. Springer, Oct. 2000. 
[44] A. Sasturkar, P. Yang, S. Stoller, and C. Ramakrishnan. Policy Analysis for Administrative Role Based Access Control. In Proceedings of the 19th Computer Security Foundations Workshop (CSFW), July 2006.

\section{APPENDIX I \\ TURING MACHINES}

A Turing Machine is denoted as $M=$ $\left(Q, \Sigma, \Gamma, \delta, q_{0}, B, F\right)$, where $Q$ is the finite set of states, $\Gamma$ is the finite set of allowable tape symbols, $B \in \Gamma$ is the blank symbol, $\Sigma \subseteq \Gamma-\{B\}$ is the set of input symbols, $\delta$ is the next move function and is a partial function from $Q \times \Gamma$ to $Q \times \Gamma \times\{L, R\}$, $q_{0} \in Q$ is the start state, and $F \subseteq Q$ is the set of final states. A nondeterministic Turing machine (NDTM) allows a finite number of choices for its next move, i.e., $\delta$ is a function from $Q \times \Gamma$ to the power set of $Q \times \Gamma \times\{L, R\}$. The first definition describes a deterministic Turing machine (DTM).

The language accepted by $M$ (denoted by $L(M)$ ) is the set of words in $\Sigma^{\star}$ that cause $M$ to enter a final state when placed justified at the left on the tape of $M$, with $M$ in state $q_{0}$, and the tape head of $M$ at the leftmost cell. A language $L$ is accepted by a DTM if and only if it is accepted by a NDTM. A language $L$ is said to be in DSPACE $(S(n))$ if there exists a DTM $M$ accepting $L$ that takes at most $S(n)$ space, where $n$ is the size of the input. Similarly, $L$ is said to be in NSPACE $(S(n))$ if there exists a NDTM $M$ accepting $L$ that takes at most $S(n)$ space. A language $L$ is said to be in PSPACE if and only if it is in DSPACE $(p(n))$ for some polynomial $p(L$ is accepted by a DTM that takes space polynomial in the size of the input). We refer the reader to [34] for more details on these and related concepts.

\section{APPENDIX II}

\section{PROOF OF LEMMA 3}

We show that URA-RC-SAP is PSPACE-hard by a reduction from the membership problem for linear bounded automata (LBA), which is known to be PSPACE-complete. A LBA is a restricted form of a Turing machine. It differs from a Turing machine in that while the tape is initially considered infinite, only a finite contiguous portion whose length is a linear function of the length of the initial input can be accessed by the read/write head.

Let $M=\left(Q, \Sigma, \Gamma, \delta, q_{0}, B, F\right)$ be any LBA that uses at most $p(n)$ space, where $n$ is the size of the input and $p$ is a degree- 1 polynomial.
(We assume that the polynomial $p(n)$ is know. However, it suffices that there exists a polynomial time algorithm to compute $p(n)$ given $n$.) We construct an RBAC system whose start state is $\gamma=$ $\langle U A, P A, R H, C A, C R, C O\rangle$ and a query $u \in r$ corresponding to the DTM $M$ so that there is an accepting computation in $M$ on an input $x$ if and only if in the RBAC system there is a sequence of RBAC states from $\gamma$ (which corresponds to input $x$ ) to $\gamma^{\prime}$ in which $u \in r$ is true.

In our construction there are two users $u_{0}$ and $u$. There is a special role $r a$ (the reader can think of this as the administrative role) and $\left(u_{0}, r a\right) \in$ $U A$. All the assigning and revoking of roles will be performed by $u_{0}$. The special user $u_{0}$ can remove users from all roles, i.e. $C R$ is equal to $\{\langle r a, R\rangle\}$, where $R$ consists of all the roles introduced in the construction except for $r a$.

Encoding $T M$ configurations. For each state $q \in$ $Q$ and $1 \leq i \leq p(n)$ we introduce a role $r_{i, a}$. For each state $q \in Q, 1 \leq i \leq p(n)$, and symbol $a$ in $\Sigma$ we introduce a role $r_{q, i, a}$. These roles are used to represent the configuration of the TM machine $M$. Additional roles will be introduced later to simulate transitions in $M$. If $u$ is assigned to the role $r_{i, a}$ then the $i$-th cell contains $a$, and the tape head is not on cell $i$. For $1 \leq i \leq p(n)$, $u$ assigned to role $r_{q, i, a}$ indicates that the tape head is on cell $i, M$ is in state $q$, and the $i$-th cell contains $a$. We add the following mutual-exclusion constraints to $C O$ to maintain the integrity of the encoding. We only add a polynomial number of constraints. We use $\left(r_{1}, r_{2}\right)$ as a shorthand for the mutual-exclusion constraint that a user cannot be members of of both $r_{1}$ and $r_{2}$ (this is equivalent to smer $\left.\left\langle\left\{r_{1}, r_{2}\right\}\right\rangle, 2\right\rangle$ in our earlier notation).

- $M$ can only be in one state at a time. We add mutual exclusion constraints of the form $\left(r_{q, i, a}, \quad r_{q^{\prime}, i, a}\right)$ for all $q \neq q^{\prime}, i$ in the range $[1, \cdots, p(n)]$ and $a \in \Sigma$.

- The tape head can only point to one location. We add mutual-exclusion constraints of the form $\left(r_{q, i, a}, r_{q, j, a^{\prime}}\right)$ for all $i \neq j$, and for all $q, a, a^{\prime}$.

- One location cannot both have the head and not have the head at the same time. We also add mutual-exclusion constraints of the form $\left(r_{i, a}, r_{q, i, a}\right)$.

- Each tape cell can only contain one symbol. 
Therefore, we add constraints of the form $\left(r_{i, a}, r_{i, a^{\prime}}\right)$ and $\left(r_{q, i, a}, r_{q, i, a^{\prime}}\right)$ for all $i, a \neq a^{\prime}$, and $q \in Q$.

Encoding the initial configuration. Assume that $M$ starts in initial state $q_{0}$, the first $n$ cells of the tape contain $a_{1}, \cdots, a_{n}$ (where $a_{i} \in \Gamma$ ), the rest of tape cells consist of blank symbols $B$, and the tape head points to the first cell. The initial state of the RBAC system has user $u$ in role $r_{q_{0}, 1, a_{1}}, r_{i, a_{i}}$ for $2 \leq i \leq n$, and $r_{j, B}$ for $n+1 \leq j \leq p(n)$.

Encoding the halting states. We use one role $r_{\text {target }}$ to be used in the query, and add the following tuples to $C A$ :

- For each $i$ in the range $[1, \cdots, p(n)], a \in \Sigma$, and accepting state $q$, add $\left\langle r a, r_{q, i, a},\left\{r_{\text {target }}\right\}\right\rangle$

This ensures that the TM $M$ enters an accepting state if and only $u$ can be assigned to $r_{\text {target }}$. The query in the URA-RC-SAP instance we are constructing is $u \in r_{\text {target }}$.

Encoding the next-move function: During each transition in $M$, two revocations and two assignments need to be done to ensure that the next configuration is correctly represented by the role memberships. We need to make these changes transactional. Therefore, we need to introduce some control roles. We will first present the construction and then explain how a state transition occurs.

We first introduce two roles $r_{b}$ and $r_{c}$. Initially, $u$ is assigned to $r_{b}$ but not $r_{c}$. The following tuple is added to $C A$ :

- $\left\langle r a, r_{c},\left\{r_{b}\right\}\right\rangle$

Suppose $\delta(q, a)$ is equal to $\left(q^{\prime}, a^{\prime}, L\right)$ (the case when $\delta(q, a)=\left(q^{\prime}, a, R\right)$ is similar). The transition $\delta(q, a)=\left(q^{\prime}, a^{\prime}, L\right)$ is modeled by doing the following.

- For each $2 \leq i \leq p(n)$, add two roles $r_{i, q^{\prime}, a^{\prime}}^{l b}$ and $r_{i, q^{\prime}, a^{\prime}}^{l c}$. Initially, $u$ is not assigned to any of these roles. We add the following mutual exclusion constraints:

- For each $2 \leq i \leq p(n)$, add constraints of the form $\left(r_{b}, r_{i, q^{\prime}, a^{\prime}}^{l c}\right)$.

- For each $2 \leq i \leq p(n)$, add constraints of the form $\left(r_{i, q^{\prime}, a^{\prime}}^{l b}, r_{c}\right)$.

- For each $2 \leq i \leq p(n)$, and each $a_{\ell} \in \Sigma$, add a role $r_{i, q^{\prime}, a_{\ell}}^{l d}$. We add the following mutual exclusion constraints:
- For each $a \leq i \leq p(n)-1$, add constraints of the form $\left(r_{i, q^{\prime}, a^{\prime}}^{l d}, r_{b}\right)$.

- We then add the following tuples to the relation $C A$ :

1) For all $2 \leq i \leq p(n)$ we add $\left\langle r a, r_{q, i, a} \cap\right.$ $\left.r_{b},\left\{r_{q^{\prime}, i, a^{\prime}}^{l b}\right\}\right\rangle$.

2) For all $2 \leq i \leq p(n)$ we add $\left\langle r a, r_{q^{\prime}, i, a^{\prime}}^{l b},\left\{r_{q^{\prime}, i, a^{\prime}}^{l c}\right\}\right\rangle$.

3) For all $2 \leq i \leq p(n)$ we add $\left\langle r a, r_{q^{\prime}, i, a^{\prime}}^{l c},\left\{r_{i, a^{\prime}}\right\}\right\rangle$.

4) For all $2 \leq i \leq p(n)$ for each $a_{\ell} \in \Sigma$ we add $\left\langle r a, r_{q^{\prime}, i, a^{\prime}}^{l c} \cap r_{i, a^{\prime}} \cap r_{i-1, a_{\ell}},\left\{r_{q^{\prime}, i, a_{\ell}}^{l d}\right\}\right\rangle$.

5) For all $2 \leq i \leq p(n)$ for each $a_{\ell} \in \Sigma$ we add $\left\langle r a, r_{q^{\prime}, i, a_{\ell}}^{l d},\left\{r_{q^{\prime}, i-1, a_{\ell}}\right\}\right\rangle$.

6) For all $2 \leq i \leq p(n)$ for each $a_{\ell} \in \Sigma$ we add $\left\langle r a, r_{q^{\prime}, i, a^{\prime}}^{l d} \cap r_{q^{\prime}, i-1, a_{\ell}},\left\{r_{c}\right\}\right\rangle$.

Transitions in the RBAC system: Suppose that at one point of computation of $M$, the tape head is at position $i$, the $i$ 'th cell contains $a$, and the current state is $q$. Then $u$ is in the role $r_{q, i, a}$. The only way to proceed in the RBAC system is to use $C A$ tuples added in step 1 to assign $u$ to $r_{q^{\prime}, i, a^{\prime}}^{l b}$. This can succeed only when $u$ is in $r_{b}$. Initially, $u$ is in $r_{b}$; and we will show that $u$ can be assigned to $r_{b}$ after a transition in $M$ has been simulated.

Once $u$ is assigned to $r_{b}$ and then to $r_{q^{\prime}, i, a^{\prime}}^{l b}$, the only way to make progress in the RBAC system is to use $C A$ tuples added in step 2 to assign $u$ to $r_{q^{\prime}, i, a^{\prime}}^{l c}$; however, because of the constraints, one has to revoke $u$ from $r_{b}$ first.

Once $u$ is assigned to $r_{b}$ and then to $r_{q^{\prime}, i, a^{\prime}}^{l c}, u$ can be assigned (using tuples added in step 3) to $r_{i, a^{\prime}}$ (after revoking $u$ from from $r_{q, i, a}$ first, due to the constraints added for tape integrity). To update roles corresponding to the $i-1$ 'th cell, let $a_{\ell}$ be the symbol on the $i-1$ 'th cell, $u$ can be assigned (using tuples added in step 4) to $r_{q^{\prime}, i, a_{\ell}}^{l d}$. Using tuples in step $5, u$ can be assigned to $r_{q^{\prime}, i-1, a_{e} l l}$. Finally, after the tape representation roles have been updated, $u$ can be assigned to $r_{c}$. Before doing this, $u$ has to be revoked from all roles of the form $r_{i, q^{\prime}, a^{\prime}}^{l b}$ because of the constraints.

To make the next state transition, $u$ must be assigned to $r_{b}$, which requires $u$ to be revoked from all roles of the form $r_{i, q^{\prime}, a^{\prime}}^{l c}$ and $r_{i, q^{\prime}, q_{e}}^{l d}$, clearing all the intermediate roles used in the simulation.

Summary: An instantaneous description (ID) of a TM $M$ is given by the contents of the tape, the 
position of the head, and the state of $M$. Given two IDs $I D_{1}$ and $I D_{2}$, we say that $I D_{1} \rightarrow_{M} I D_{2}$ if $I D_{2}$ follows from $I D_{1}$ by one move of the $M$. Given an input $x$, let $I D_{0}, \cdots, I D_{n}$ be a sequence of IDs such that $I D_{0}$ corresponds to the input $x$, $I D_{i} \rightarrow_{M} I D_{i+1}$, and $I D_{n}$ has an accepting state (the sequence is an accepting computation to the string). Let $\gamma_{0}$ be the RBAC-state that corresponds to $I D_{0}$. Each move of the Turing machine can be emulated by the RBAC scheme in a number of steps; let this constant be $c$. Therefore, there exists a sequence of RBAC-states $\gamma_{0}, \cdots, \gamma_{c n}$ such that $\gamma_{c i}$ encodes $I D_{i}, \gamma_{i} \rightarrow \gamma_{i+1}$, and in $\gamma_{c n} u$ is in a role $r_{q, i, a}$ such that $q \in F$. And then $u$ can be assigned to $r_{\text {target }}$. Hence an accepting computation in $M$ has a corresponding sequence in the RBAC system.

Our discussion of state transitions in the RBAC system above show that state changes in the RBAC systems correspond to computations. If a user $u$ is assigned role $r_{q, i, a}$ in some state in the RBAC system, there is a corresponding computation in the TM $M$ that reaches state $q$ and has the head on the $i$ 'th position with the symbol $a$ in the $i$ 'th position.

Therefore, there is an accepting computation in $T M$ on an input $x$ if and only if in there is a sequence of RBAC-states from $\gamma$ (which corresponds to input $x$ ) to $\gamma_{m}$, where $u$ is in role $r_{\text {target }}$. This proves the result.

\section{APPENDIX III \\ PROOF FOR LEMMA 6}

Proof: We reduce 3SAT to the URA-RC-SAP, which proves that URA-RC-SAP is NP-hard. Let $f=c_{1} \wedge \cdots \wedge c_{m}$ be an instance of 3SAT, and let $p_{1}, \ldots, p_{n}$ be the propositional variables in $f$. We associate a role, $r_{f}$, with the formula $f$. We now construct an instance of URA-RC-SAP [ $C A$ (conjunctive), $C R=\{\}, C O=\{\}]$ so that a user becomes a member of $r_{f}$ if and only if $f$ is satisfiable.

In addition to $f, \mathcal{R}$ contains roles $c_{1}, \ldots, c_{m}$ corresponding to the clauses and roles $p_{1}, \ldots, p_{n}$ corresponding to the propositional variables, plus a role $t$ that will be used for signaling, as will be explained shortly. We construct $C A$ so as to allow $u$ to be assigned to any combination of the $p_{i}$ 's. Once this is done, $C A$ will permit $u$ to be assigned to role $t$, signaling that a second phase has begun in which $u$ can be added to role $c_{j}$ just in case $u$ 's assignment to the $p_{i}$ 's represents a truth assignment that makes clause $c_{j}$ true. More precisely, $C A=\left\{\left\langle a, \neg t,\left\{p_{1}, \ldots, p_{n}\right\}\right\rangle,\langle a\right.$, true,$\{t\}\rangle,\left\langle a, c_{1} \cap\right.$ $\left.\left.\cdots \cap c_{m}, f\right\rangle\right\} \cup\left\{\left\langle a, t \cap p_{i},\left\{c_{j} \mid 1 \leq j \leq\right.\right.\right.$ $m \wedge p_{i}$ appears positively in $\left.\left.c_{j}\right\}\right\rangle \mid 1 \leq i \leq$ $n\} \cup\left\{\left\langle a, t \cap \neg p_{i},\left\{c_{j} \mid 1 \leq j \leq m \wedge\right.\right.\right.$ $p_{i}$ appears negatively in $\left.\left.\left.c_{j}\right\}\right\rangle \mid 1 \leq i \leq n\right\}$ and $C R=\emptyset$.

We now consider an instance of URA-RC-SAP in which $A_{T}, U A, P A$ and $R H$ are empty, and show that it is true if and only if $f$ is satisfiable. If the formula $f$ is satisfiable, it is easy to see that $a$ can add $u$ to role $f$ by first adding $u$ to the role $p_{i}$ if the propositional variable $p_{i}$ is true in the solution to $f$, then adding $u$ to $t$, and then adding $u$ to each $c_{j}$ and finally to $f$.

Conversely, if the problem instance is true, then at some point $u$ must be added to role $t$. Since $u$ cannot be removed from $t, u$ 's assignment to the $p_{i}$ roles at that time enables $u$ to be added to each role $c_{j}$. By defining the propositional variables $p_{i}$ to be true if and only if the role $p_{i}$ contains $u$ at that time, we get an assignment that makes at least one literal true in each clause $c_{j}$.

\section{APPENDIX IV \\ PROOF FOR LEMMA 7}

Proof: We reduce monotone 3SAT to the problem. Monotone 3SAT is a special case of 3SAT where all literals in a clause are either all positive or all negative; monotone 3SAT is known to be NPcomplete [14].

Let $e=c_{1} \wedge \ldots \wedge c_{l} \wedge \overline{c_{l+1}} \wedge \ldots \wedge \overline{c_{n}}$ be an instance of monotone 3SAT, where $c_{1}, \ldots, c_{l}$ are the clauses with only positive literals, and $\overline{c_{l+1}}, \ldots, \overline{c_{n}}$ are the clauses with only negative literals. Let $p_{1}, \ldots, p_{k}$ be all the propositional variables that appear in $e$, and each $c_{i}=p_{i_{1}} \vee p_{i_{2}} \vee p_{i_{3}}$ and each $\overline{c_{j}}=\neg p_{j_{1}} \vee \neg p_{j_{2}} \vee$ $\neg p_{j_{3}}$. We produce a corresponding URA-RC-SAP instance for $R B A C_{\text {assign, nonegation }}$ as follows.

Corresponding to each propositional variable, $p_{i}$, we have a role, $r_{i}$. We also have a role, $r_{c_{i}}$, corresponding to each positive clause $c_{i}$ in $e$. In addition, we have the roles $r$ and $a$. We assign a user $u_{0}$ to $a$, that is, $\left\langle u_{0}, a\right\rangle \in U A$. The role $a$ is an administrative role, and appears as the first component of every entry in $C A$. We first add the tuple $\left\langle a, r_{c_{1}} \wedge \ldots \wedge r_{c_{l}}, r\right\rangle$ to $C A$. Corresponding to each positive clause $c_{i}=p_{i_{1}} \vee p_{i_{2}} \vee p_{i_{2}}$, we add 
the three entries $\left\langle a, r_{i_{1}}, r_{c_{i}}\right\rangle,\left\langle a, r_{i_{2}}, r_{c_{i}}\right\rangle,\left\langle a, r_{i_{3}}, r_{c_{i}}\right\rangle$ to $C A$. If $c_{i}$ has fewer than 3 literals, then we only add such entries to $C A$ that correspond to the literals in $c_{i}$. Clearly, the $C A$ so constructed has no negation in the preconditions of its entries, and each precondition is a conjunction of roles. We capture the negative clauses in $e$ using entries in $C O$. For each negative clause $\overline{c_{j}}=\neg p_{j_{1}} \vee \neg p_{j_{2}} \vee \neg p_{j_{3}}$, we add the constraint smer $\left\langle\left\{r, r_{j_{1}}, r_{j_{2}}, r_{j_{3}}\right\}, 4\right\rangle$. If $\overline{c_{j}}=\neg p_{j_{1}} \vee \neg p_{j_{2}}$ (that is, has only 2 literals), then we add the constraint smer $\left\langle\left\{r, r_{j_{1}}, r_{j_{2}}\right\}, 3\right\rangle$ to $C O$, and if $\overline{c_{j}}=\neg p_{j_{1}}$ (has only one literal), then we add the constraint smer $\left\langle\left\{r, r_{j_{1}}\right\}, 2\right\rangle$ to $C O$.

We ensure that the user $u$ that is specified in the query $q$ is not a member of $r$ or any $r_{c_{i}}$ in the start-state $\gamma$. We now assert that there exists a reachable state in which $u$ is a member of $r$ if and only if $e$ is satisfiable. The reason is that the only way $u$ can become a member of $r$ is by $u_{0}$ successfully exercising the only entry in $C A$ that has $r$ as the target role (last component of the tuple in $C A$ ). This is possible if and only if $u$ already satisfies the role-memberships as specified in the precondition, and assigning $u$ to $r$ does not violate any of the entries in $C O$. More formally, for the "if" part, assume that $e$ is satisfiable. Then there is some truth-assignment that makes $e$ true. We use the truth-assignment as the user-role assignment in $\gamma$ for $u$. That is, if the propositional variable $p_{i}$ is true in the truth-assignment that makes $e$ true, then $\left\langle u, r_{i}\right\rangle \in U A$. Now, $u_{0}$ will be able to assign $u$ to a role $r_{c_{i}}$ if and only if $\left\langle u, r_{i_{1}}\right\rangle \in U A,\left\langle u, r_{i_{2}}\right\rangle \in U A$ or $\left\langle u, r_{i_{3}}\right\rangle \in U A$, where $c_{i}=p_{i_{1}} \vee p_{i_{2}} \vee p_{i_{3}}$, and none of the entries in $C O$ is violated. An entry in $C O$ is violated if and only if $u$ is a member of all roles other than $r$ in the set of the roles in a constraint. This is the case if and only if the corresponding negative clause is false, which is impossible given the assumption that $e$ is satisfiable.

For the "only if" part, assume that there exists a reachable state in which $u$ is a member of $r$. We use the role-memberships of $u$ in the roles $r_{1}, \ldots, r_{k}$ in the start-state $\gamma$ as our truth-assignment for $e$. That is, if $\left\langle u, r_{i}\right\rangle \in U A$, then we set the corresponding propositional variable, $p_{i}$, to be true. Given that $u$ can eventually be assigned to $r$, we know that $u$ can be assigned to every $r_{c_{i}}$ in $\gamma$. Therefore, each positive clause is true. Furthermore, given that in the final action, we can assign $u$ to $r$, we know that no entry in $C O$ is violated. Consider the constraint smer $\left\langle\left\{r, r_{i_{i}}, r_{i_{2}}, r_{i_{3}}\right\}, 4\right\rangle$. This constraint would disallow $u_{0}$ from assigning $u$ to $r$ if and only if $u$ is already a member of all of $r_{i_{1}}, r_{i_{2}}$ and $r_{i_{3}}$. As this is not the case, we know that every negative clause evaluates to is true. 